\title{
Scalp hair repigmentation in the penumbral region of radiotherapy- a case series
}

\begin{abstract}
Introduction: Hair colour is determined by varying ratios of black-brown eumelanin and reddish-brown/reddish-yellow pheomelanin. Hair colour change has been reported with cancer therapies. Radiotherapy (RT) usually causes temporary epilation to permanent alopecia. A change in hair colour following radiation is rare and usually results in depigmentation. There has only been one other case reported of repigmentation after RT.

Cases: We present five cases of changes to scalp hair pigmentation in the penumbral region in patients treated with volumetric modulated arc therapy (VMAT) for skin cancer. Five treated areas across four patients involved repigmentation from grey to black, and there was one case of depigmentation from brown to grey. The latter occurred during immunotherapy administration. For the two cases where recalculation of the dosimetry was possible for three areas, repigmentation changes occurred at an average mean dose of 16 Gy $(9.3-26$ Gy) in an average of $26(25$ - 27) fractions; that is, 0.6 Gy per fraction at five fractions per week.

Discussion: This series of six areas in five patients of hair colour change in the penumbral region of VMAT to the scalp for skin cancer is the first report of this phenomenon. Repigmentation of scalp hair with RT is rare. Even though rare, the potential for hair colour change may need to be part of the informed consent discussion in patients contemplating this treatment.
\end{abstract}

Keywords: hair, radiotherapy, skin cancer, pigmentation, hair colour, scalp, volumetric modulated arc therapy
Volume 7 Issue 5 - 2020

\author{
Shimon Prasad,' Nicole Dougheney,' \\ Angela Hong, 1,2 John Flood,,4 David Wong, ${ }^{5}$ \\ Elizabeth Paton, ${ }^{6}$ Gerald B Fogarty, 2,4 \\ 'GenesisCare, Mater Sydney Hospital, North Sydney NSW \\ 2065, Australia \\ ${ }^{2}$ Melanoma Institute Australia, The University of Sydney, North \\ Sydney NSW 2060, Australia \\ ${ }^{3}$ Sydney Adventist Hospital, Wahroonga NSW 2076, Australia \\ ${ }^{4}$ St Vincent's Private Hospital, Darlinghurst NSW 2010, Australia \\ ${ }^{5}$ Eastern Suburbs Dermatology, Bondi Junction NSW 2022, \\ Australia \\ ${ }^{6}$ Melanoma and Skin Cancer Trials Group, Monash University, \\ Clayton VIC 3800, Australia
}

Correspondence: Gerald B Fogarty, GenesisCare Radiation Oncology, Mater Hospital, Rocklands Rd, Crows Nest NSW 2065, Australia, Tel +6I 29458 8050, Fax +6I 29929 2687, Email Gerald.Fogarty@genesiscare.com

Received: September 13,2020 | Published: September 28, 2020

\section{Introduction}

Hair colour is important for appearance and hair colouring is a well-established industry. Hair colour is determined by varying ratios of black-brown eumelanin and reddish-brown/reddish-yellow pheomelanin. ${ }^{1}$ Greying hair, also called achromotrichia, indicates a lack of pigment which can happen naturally with ageing. Hair repigmentation is when greying hair becomes coloured once again. Hair colour change has been reported with cancer therapies. Repigmentation is documented following untargeted chemotherapy for breast cancer, ${ }^{2}$ anti-PD-1 or anti-PD-L1 therapy for non-small cell lung cancer ${ }^{3}$ and following imatinib. ${ }^{4}$ Radiotherapy (RT) usually causes temporary epilation to permanent alopecia depending on the total dose, fractionation, type of hair and how close the radiation target is to the hair bearing area. Hair colour change following radiation is rare and usually involves depigmentation. ${ }^{5}$ We present five cases with six areas of scalp hair pigmentation change in the penumbral region when these patients were being treated with volumetric modulated arc therapy (VMAT) for skin cancer. Five areas in four patients experienced repigmentation, and one had depigmentation.

\section{Cases} 1.

Hair and dose characteristics for all cases are summarised in Table .

Table I Patient characteristics

\begin{tabular}{llllll}
\hline Patient & Age (Yrs) & Comments & VMAT for & Script dose(Gy)/\# & Hair change \\
\hline 1 & 51 & ESFC & $50 / 25$ & Repig \\
2 & 84 & MCC & $50 / 25$ & Repig \\
3 & 62 & ESFC & $55 / 25$ & Repig \\
4 & 81 & $\begin{array}{l}\text { Immunosuppressed } \\
\text { with PMR }\end{array}$ & ESFC & $48.7 / 27$ & Repig \\
5 & 71 & On Immunotherapy & MM & $30 / 10$ & Depig \\
Average & 69.8 & & & $51 / 26(25-27)$ (Exclude 5) &
\end{tabular}

Yrs, years; VMAT, volumetric modulated arc therapy; ESFC, extended skin field cancerisation; MCC, merkel cell carcinoma; PMR, polymyalgia rhuematica; MM, malignant melanoma; SIB, simultaneous integrated boost; Gy, Gray; \#, fraction; Repig, repigmentation; Depig, depigmentation 


\section{Case I}

A 51-year old Caucasian male with no previous medical history, apart from multiple skin cancers requiring surgery, was referred for VMAT for extended skin field cancerisation (ESFC) of the forehead. The patient had male pattern baldness and multiple biopsy-confirmed symptomatic basal cell carcinomas amongst a field of in-situ cancer on the forehead. He was treated with 50 Gy in 25 fractions to a field measuring 20 centimetres $(\mathrm{cm})$ by $15 \mathrm{~cm}$ with our published technique. ${ }^{6}$ Two circular areas within this field measuring $3 \mathrm{~cm}$ and $5 \mathrm{~cm}$ in diameter respectively (Figures $1 \mathrm{~A} \& \mathrm{~B}$ ) were taken to a dose of $60 \mathrm{~Gy}$ in 25 fractions with a simultaneous boost technique (SIB). This technique is delivered with a substantial tangential component ${ }^{7}$ to avoid dose to the brain. The treatment plan had an appreciable penumbral component in the surrounding scalp. The patient finished the treatment on time over five weeks with no breaks and with the expected toxicities of tumorlysis and desquamation, which were cared for with conservative measures until healed. He presented for routine review nine months later with no sign of recurrence infield of invasive or in situ cancer. Although satisfied with his overall treatment, he complained of areas of hair close to the treatment field having reverted to the colour he had in his younger days. These areas were measured by use of a template. ${ }^{8}$ The template marks were then superimposed over his original planning scan. The dose of radiation the pigmented areas had received was then calculated. Dose volume histograms (DVHs) were calculated for these areas (Figures $1 \mathrm{C}-\mathrm{H})$. Dosimetry of the penumbral areas where the hair colour change was noted is detailed in Table 2.

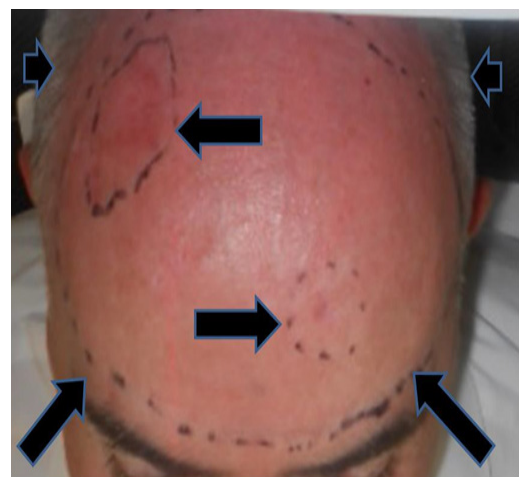

Figure IA Planning photo. Long horizontal arrows indicate the areas of simultaneous integrated boost. Diagonal arrows indicate field edge. Short horizontal arrows indicate nearby but out of field areas of skin, bearing grey hair. These border the treated bare scalp that is exhibiting male pattern baldness.

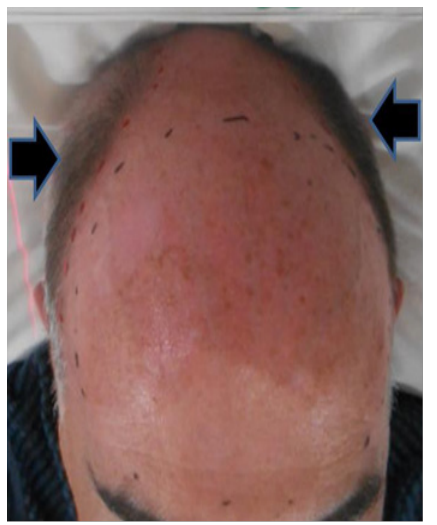

Figure I B Nine months after treatment. There is no sign of disease. However horizontal arrows indicate the out-of-field areas of hair-bearing skin that that have repigmented from grey to black. Black dotted line shows the previous treatment field.

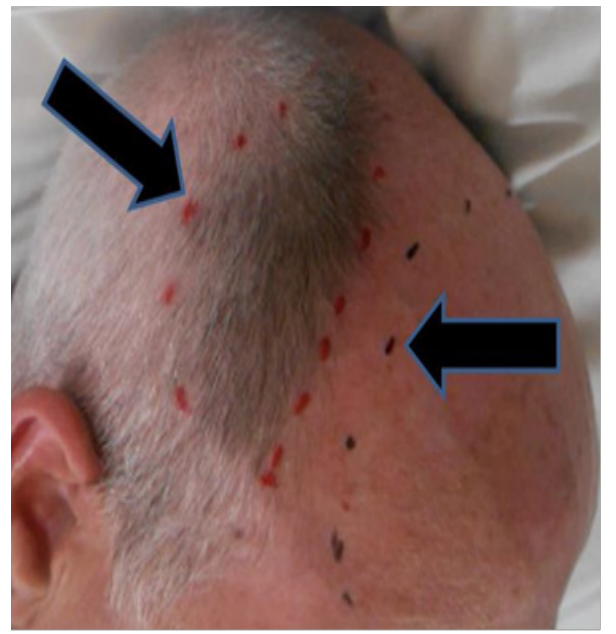

Figure IC Nine months after treatment. Photo taken from right lateral position. Horizontal arrow and black dotted line show previous treatment field. Diagonal arrow and red dotted line indicate out of field hair that has re-pigmented.

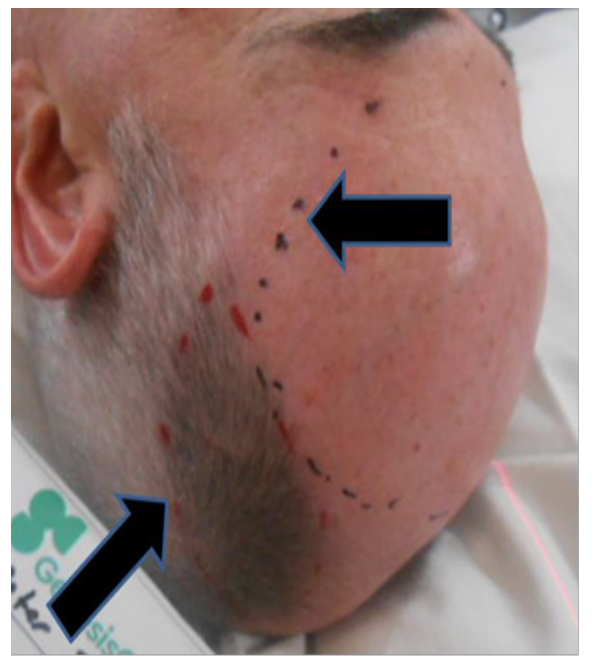

Figure ID Nine months after treatment. Photo taken from left lateral position. Horizontal arrow and black dotted line show previous treatment field. Diagonal arrow and red dotted line indicate out-of-field hair that has repigmented.

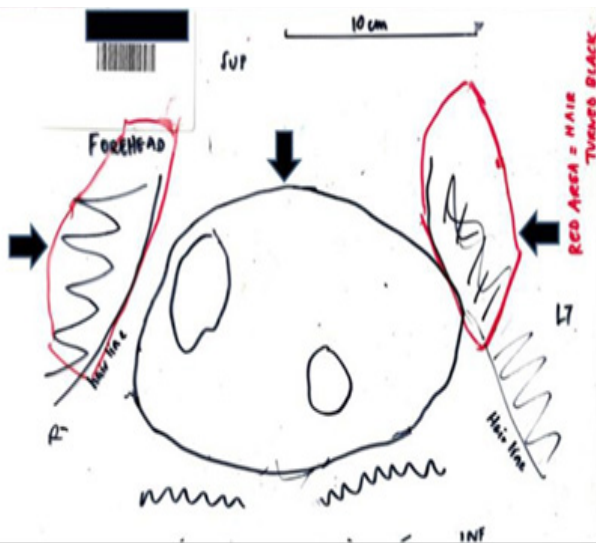

Figure IE Nine months after treatment. Photo of template made of Case I. Orientation of the template is that the superior border is the vertex of scalp; inferior border is at the level of the orbits. Vertical arrow shows previous treatment field. Horizontal arrows show areas of out-of-field hair that has repigmented. 


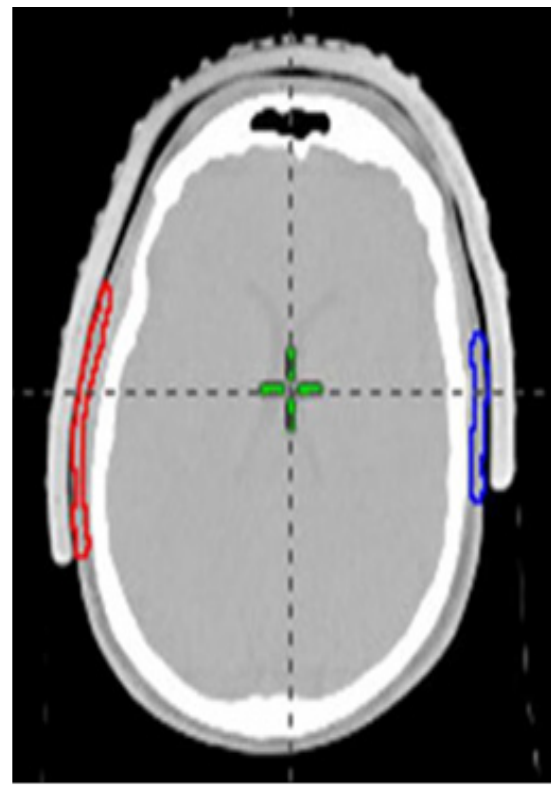

Figure IF Template marks of repigmented hair were superimposed on the patient's original planning scan. Red volume is right sided and blue volume is left sided. Axial slice.

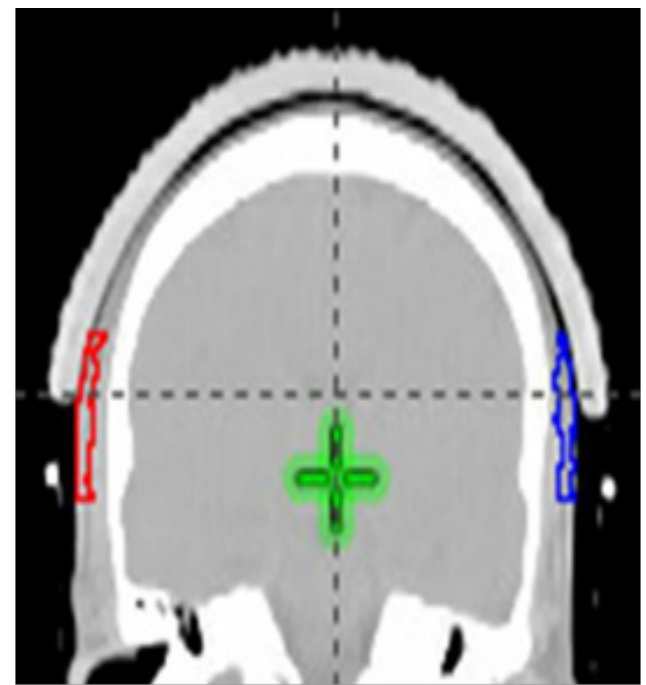

Figure IG Template marks of repigmented hair were superimposed on the patient's original planning scan. Red volume is right sided and blue volume is left sided. Coronal slice.

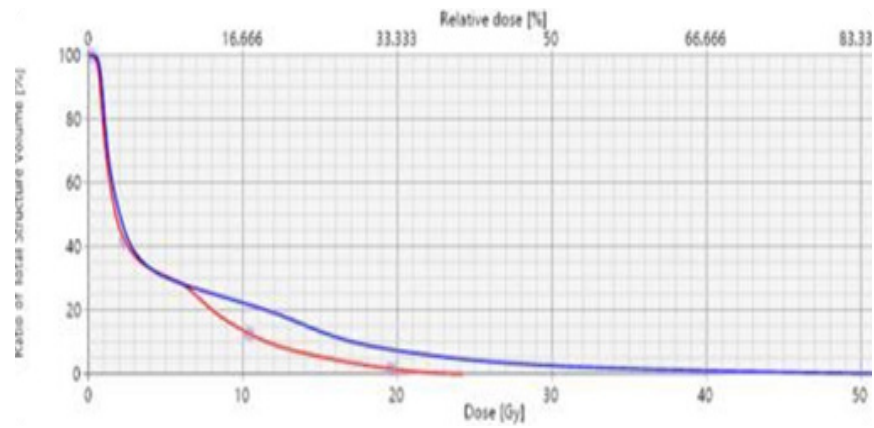

Figure IH Dose volume histograms (DVHs) were calculated for these volumes from the original dosimetry. Red line shows right repigmented hair dose characteristics. Blue line shows left repigmented hair dose characteristics.
Table 2 Dose characteristics to contoured penumbral regions

\begin{tabular}{lllll}
\hline Patient & Hair change & Max (Gy) & Mean (Gy) & Min(Gy) \\
\hline I - Right & Repig & 24 & 12.3 & 0 \\
I - Left & Repig & 52 & 9.3 & 0.3 \\
2 & Repig & 52 & 26 & 2.8 \\
Repig Ave & & 43 & $16(9.3-26)$ & 1 \\
5 & Depig & 21 & 15 & 5 \\
All Ave & & 38 & 16 & 3
\end{tabular}

Gy, Gray; Repig, repigmentation; Depig, depigmentation;Ave, averages

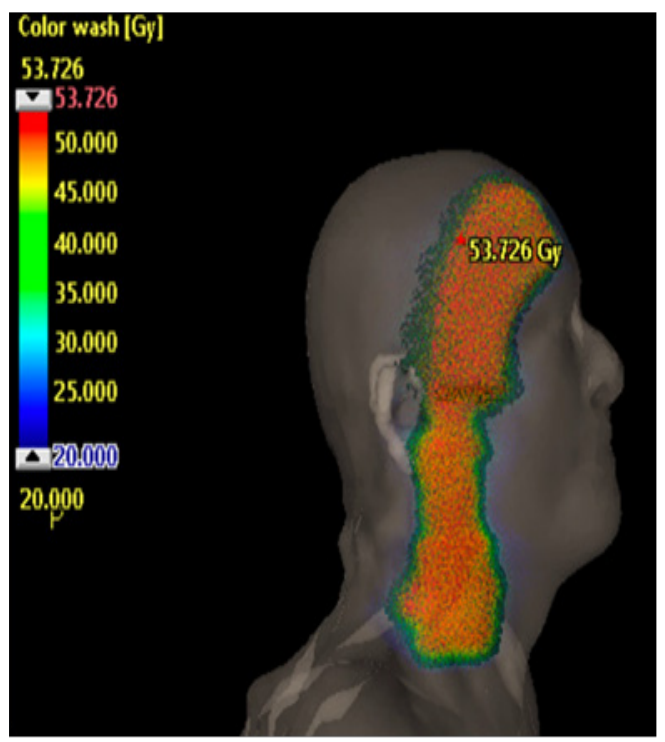

Figure 2 Patient photos of Case 2. (A) Dosimetry of Case 2.

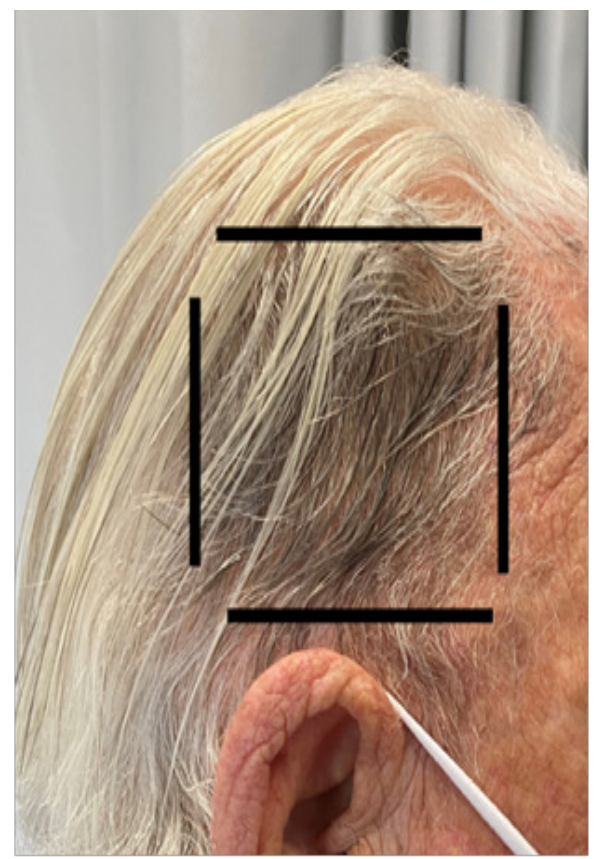

Figure 2B Hair repigmentation covers an area above the pinna. 


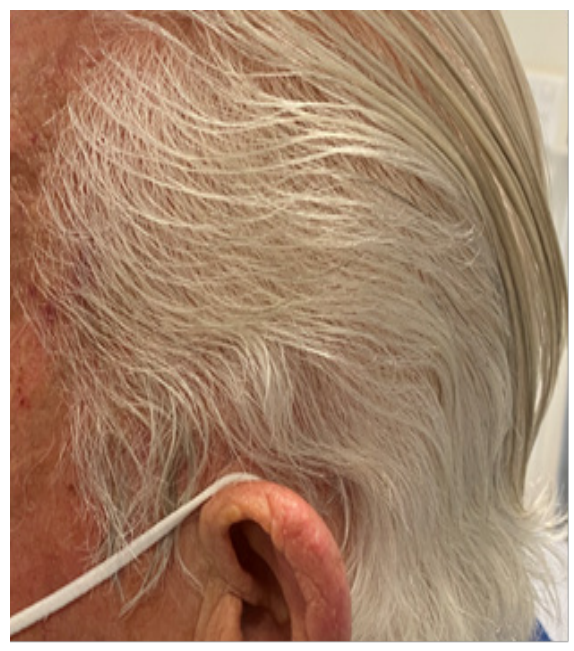

Figure 2C As expected, the unirradiated contralateral side has no hair repigmentation.

\section{Case 2}

An immunocompetent 84-year-old Caucasian male was referred for VMAT following excision of a Merkel cell carcinoma on the right temple. He was treated with 50 Gy in 25 fractions and finished the treatment on time over five weeks with no breaks. Figure 2A shows the patient's planning scan and dosimetry. At the patient's six-month review, he was noted to have hair repigimentation that covered an area above the pinna that would have been in the penumbra (Figure $2 \mathrm{~B})$. Figure $2 \mathrm{C}$ shows the normal appearance on the unirradiated contralateral temple. The dosimetry of penumbral areas where the hair colour change was noted is detailed in Table 2 .

\section{Case 3}

An immunocompetent 62-year old Caucasian male was referred for VMAT for extended skin field cancerisation (ESFC) of the scalp. He had no previous medical history except for multiple skin cancers treated with surgery. His scalp exhibited male pattern baldness and had multiple biopsy-confirmed in-situ squamous cell carcinomas. He was treated with $55 \mathrm{~Gy}$ in 25 fractions to a wide field. He finished the treatment on time over 5 weeks with no breaks. On review fourteen months after RT, a darkened $4 \mathrm{~cm}$ thick strip on the posterior scalp that wrapped around to both temples was noticed (Figure 3). Retrospective dosimetry could not be undertaken.

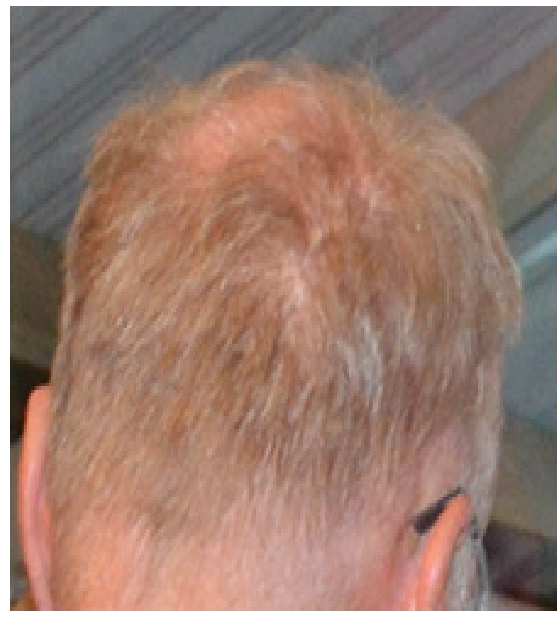

Figure 3 Patient photos of Case 3. (A) Case 3 prior to RT.

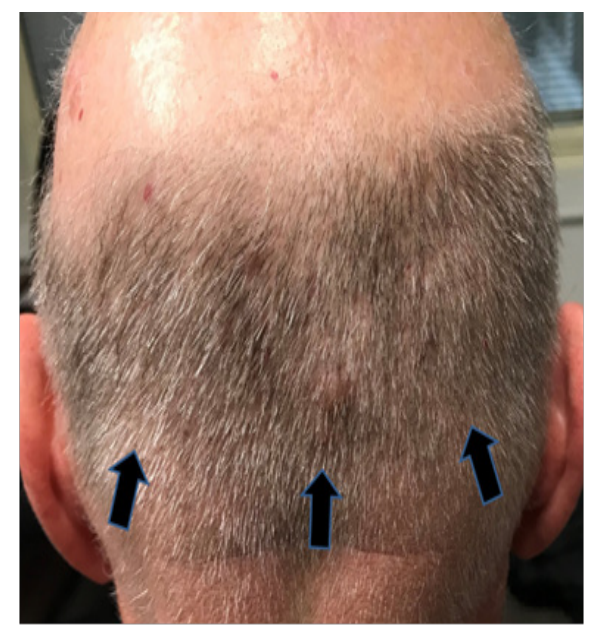

Figure 3B Case 3 fourteen months post RT.Arrows show the darkened strip. Posterior view.

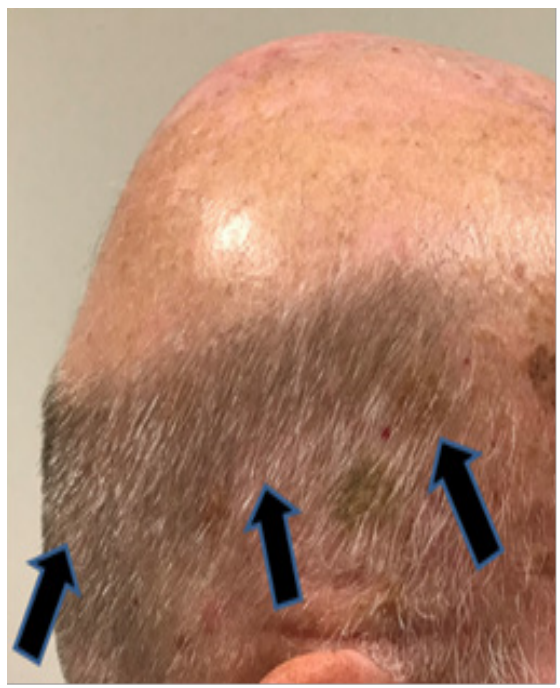

Figure 3C Case 3 fourteen months post RT. Right lateral view

\section{Case 4}

An 81-year old immune competent Caucasian male presented for consideration of VMAT for ESFC of the scalp. His relevant history included polymyalgia rheumatica (PMR) for several years requiring intermittent steroids, multiple scalp skin cancers previously treated with surgery and male pattern baldness. He was treated with $48.7 \mathrm{~Gy}$ in 27 fractions and terminated treatment before the prescribed 54 Gy due to the development of painful moist desquamation. Six months after RT during routine review, he was already noted to have an area of black hair similar to his original hair colour before going grey. This rim of dark hair corresponded to the penumbral low dose radiotherapy region. Retrospective dosimetry could not be carried out (Figure 4).

\section{Case 5}

A 71-year old Caucasian male with metastatic melanoma required whole brain radiotherapy (WBRT). He was initially on combination immunotherapy with nivolumab and ipilimumab which was changed two months after WBRT to single agent nivolumab. He was treated with a hair-sparing protocol. ${ }^{9}$ The trial specific prescription delivered $30 \mathrm{~Gy}$ in 10 fractions to the whole brain as well as $40 \mathrm{~Gy}$ in 10 fractions via a SIB technique to two MRI detected asymptomatic brain metastases. The study delivered a lower dose to contoured 
superior (supscalp) and inferior (infscalp) scalp regions to try to spare scalp hair. The trial specific inferior scalp region ("inf scalp") was a volume that included a hair bearing region that extended from a line 2 $\mathrm{cm}$ above the pinna to the hair line at the nape of the neck. The patient remained on nivolumab and $7.5 \mathrm{mg}$ of daily prednisolone throughout WBRT treatment. Figure 5 shows the hair colour prior to therapy. The original "inf scalp" volume (Figure 5B) received a maximum of 21 Gy, a minimum of $0.5 \mathrm{~Gy}$ and a mean dose of $11.7 \mathrm{~Gy}$. One month post treatment, there was no change in hair colour but epilation infield was apparent (Figure 5C). Hair below the arrows is the patient's original hair colour prior to WBRT which retained its colour. At four months post WBRT, there was depigmentation of both the sup and inf scalp volumes. In order to be more precise about the hair colour change inferiorly, a new contour was created called "new infscalp". This contour replicated the infscalp volume except that the lower border was on the line of the hair colour change, aligning it to the end of the WBRT field (Figure 5D). The dose was then recalculated from the original plan and a new dose volume histogram was produced (Figure 5F). The new "infscalp" volume (Figure 5B) received a maximum of $21 \mathrm{~Gy}$, a minimum of $5 \mathrm{~Gy}$ and mean dose of $15 \mathrm{~Gy}$ (Table 2). Dosimetry of penumbral areas where the hair colour change was noted is detailed in Table 2.

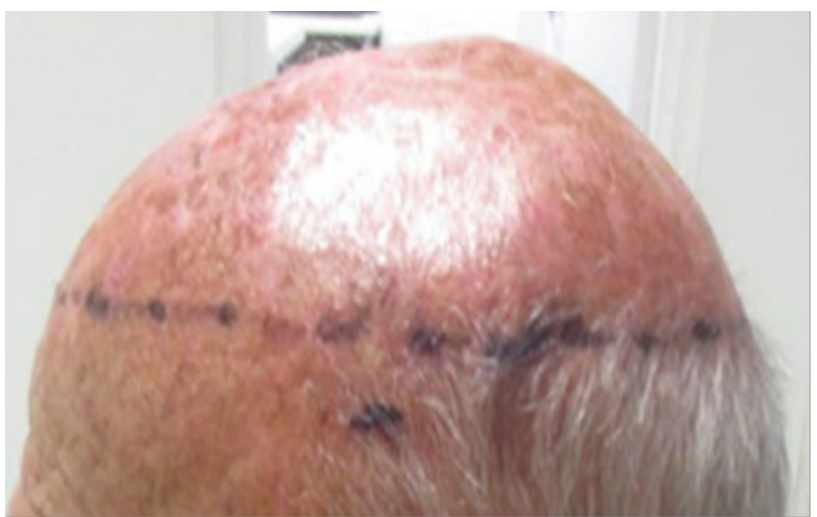

Figure 4 Patient photos of Case 4. (A) Planning photo. Note the hair is all grey prior to RT.

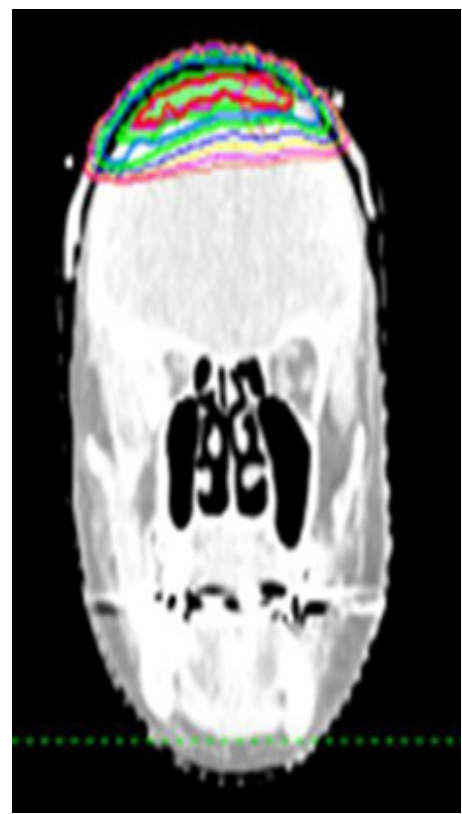

Figure 4B Coronal plane of planning CT.The outermost isodose line is $27 \mathrm{~Gy}$. The colour change is outside this volume.

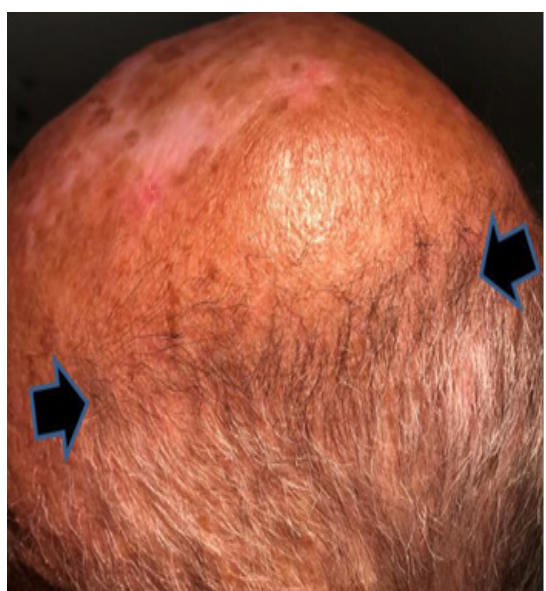

Figure 4C Photo two years after RT. The arrows delineate a persisting repigmentation of the hair that has grown back following RT.

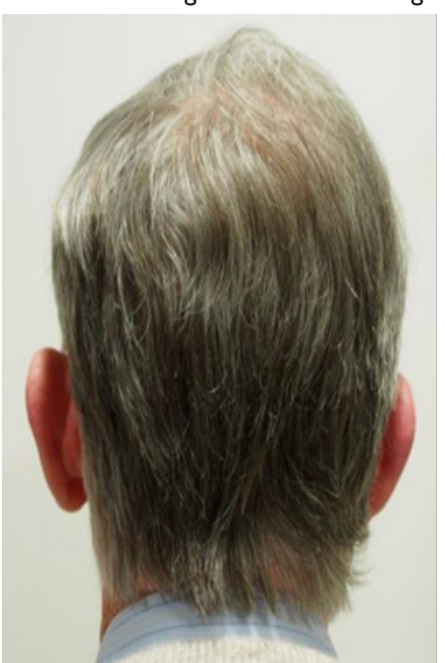

Figure 5 Patient with metastatic melanoma on immunotherapy who also participated in the Hair Spare WBRT study. (A) Photo taken prior to WBRT hair sparing trial. ${ }^{9}$

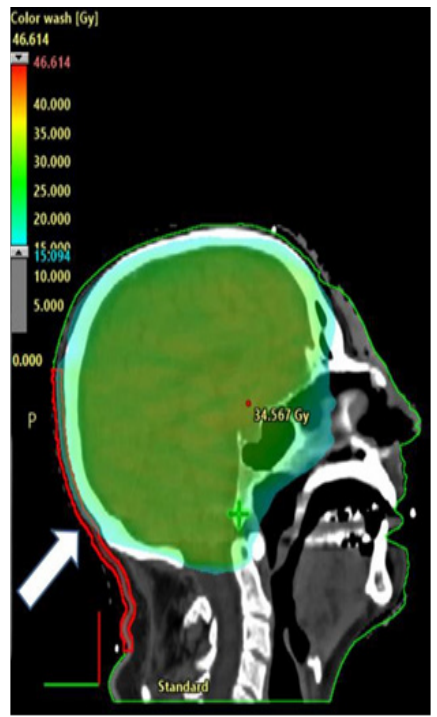

Figure 5B Dosimetry on the midline sagittal planning scan from dose wash of I5 Gy of the Hair Spare protocol. ${ }^{9}$ Note the white arrow showing the trial specific infscalp volume which goes inferiorly to the hair line at the nape of the neck. 


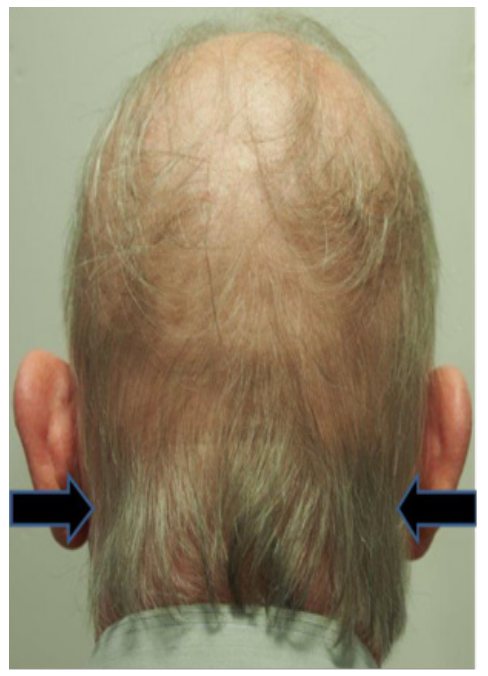

Figure 5C One month post hair-sparing WBRT on trial. Arrows denote the lower level of the WBRT field. This is the level of the new infscalp volume.

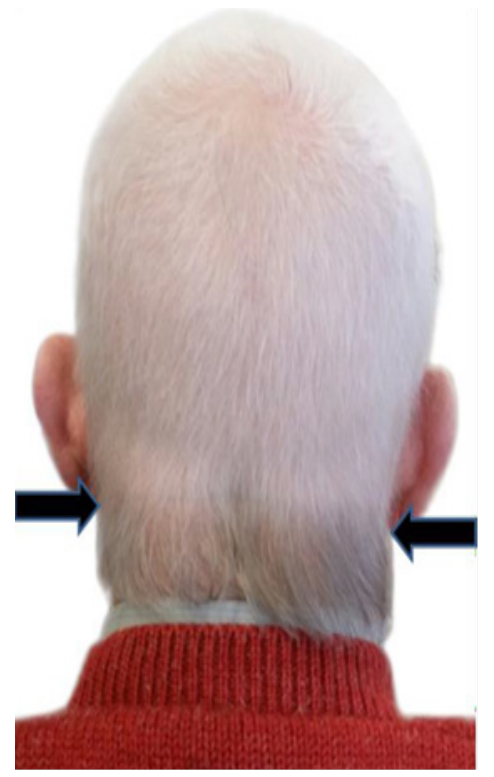

Figure 5D Four months post hair-sparing WBRT on trial. There is depigmentation of both the sup and inf scalp volumes. Arrows denote the lower level of the WBRT field. Note the change in hair colour above this line.

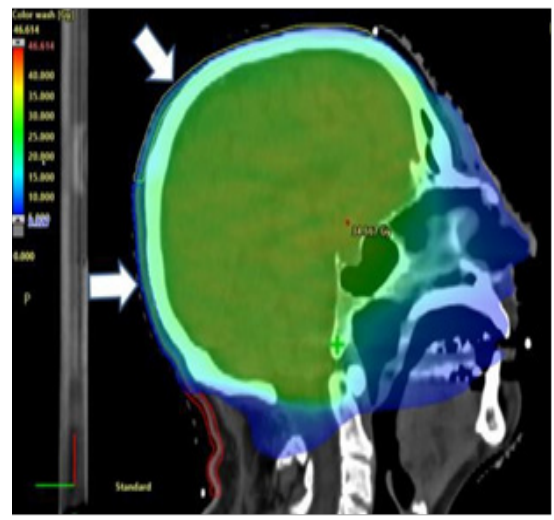

Figure 5E Dosimetry on the midline sagittal planning scan from dose wash of $5 \mathrm{~Gy}$ of the Hair Spare protocol. Note the diagonal white arrow showing the trial specific supscalp volume. The horizontal white arrow shows the new infscalp volume which goes inferiorly only to the level of the change in hair colour.

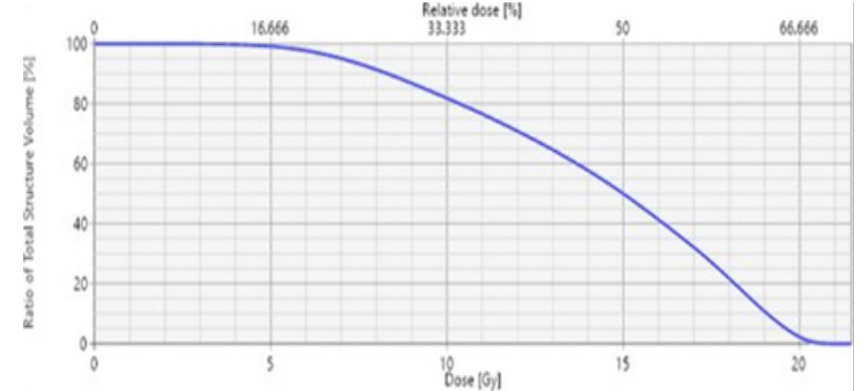

Figure 5F DVH of "new infscalp" contour.

\section{Discussion}

We present six areas in five patients with changes in hair colour in the penumbral region following VMAT radiotherapy to the scalp. Five areas in four cases involved repigmentation from grey to black and the other case was depigmentation from brown to grey. The latter occurred during immunotherapy administration. For the two cases where dosimetry calculation was possible for three affected areas of repigmentation, changes happened at an average mean dose of $16 \mathrm{~Gy}$ $(9.3-26 \mathrm{~Gy})$ in an average of 26 (25 - 27) fractions; that is $0.6 \mathrm{~Gy}$ per fraction at five fractions per week. Hair colour is determined by varying ratios of black-brown eumelanin and reddish- brown/reddishyellow pheomelanin ${ }^{1}$. These proteins are synthesized by melanocytes which arise from megaloblasts contained in the bulge area of the outer root sheath of hair follicles. ${ }^{10}$ Hair is actively pigmented in the anagen phase. ${ }^{11}$ The anagen phase in the human adult scalp can last for many years. Achromotrichia indicates a lack of pigment being produced and can happen naturally with aging. Achromotrichia may also be triggered by the accumulation of hydrogen peroxide found in patients suffering from vitiligo, ${ }^{12}$ and is associated with increased UV exposure, stress, malnutrition like vitamin B12 deficiency, thyroid deficiencies, smoking and some genetic factors such as albinism. Grey hair in the non-balding may grow more quickly than pigmented hair. ${ }^{13}$

Cancer therapies are known to cause hair colour change. Repigmentation was investigated by Watanabe et al. ${ }^{2}$ following chemotherapy for breast cancer. Of 1478 patients, 73 (5.3\%) became darker, 525 (38.4\%) became more grey or whiter, and $717(52.5 \%)$ had no change. Hair repigmentation has been reported with anti-PD-1 or anti-PD-L1 therapy for non-small cell lung cancer by Rivera et al. ${ }^{3}$ Thirteen patients experienced diffuse darkening of the hair; one had black patches between white hairs. Those with hair colour change had good oncological outcomes, responding well to therapy. The authors suggested that hair repigmentation may be a good response marker. The tyrosine kinase inhibitor imatinib has recently been shown to reverse the greying process. ${ }^{4}$ Radiation usually causes temporary epilation to permanent alopecia depending on the total dose, fractionation, hair type and how close the radiation target is to the hair-bearing area. For the ionising radiation effects on hair, ICRP 85 sets the dose at 7 Gy in a single exposure for permanent alopecia and a maximum of 3 Gy to avoid temporary epilation. ${ }^{14}$ Fractionation appears to allow dose escalation without increasing alopecia. Doses as low as 2 Gy in four fractions can cause temporary anagen alopecia. ${ }^{15}$ Lawenda et al. ${ }^{16}$ graded the degree of alopecia on each region of the scalp (Grade 1, none; Grade 2, minimal; Grade 3, moderate; and Grade 4, severe). In 26 patients they found that permanent alopecia correlated significantly with the follicle dose only $(\mathrm{p}<0.001)$. They suggested a follicle dose of 25 Gy was associated with a $20 \%$ risk of permanent Grade 3 or greater alopecia when using 1.5-2.0 Gy fractions using megavoltage radiotherapy (MV). Hair colour change following radiation is less often reported. Repigmentation is very rare. Alexander ${ }^{5}$ reported one 
case in 1963 after irradiation for benign disease to 3 Gy over three fractions given second daily with orthovoltage.

The dose of 16 Gy occurs a few times in the hair literature and also in our work. ${ }^{9}$ With $\mathrm{MV}$, if the dose of radiation to a depth of five millimetres is kept under 16 Gy with fractionated therapy, which is the approximate lethal dose for hair follicles, the incidence of radiationinduced alopecia can be avoided or markedly decreased. ${ }^{17} \mathrm{~A}$ recent prospective trial reported by our group, attempting hair preservation using a VMAT hair-sparing protocol during WBRT, found that the rate of alopecia at four weeks was decreased when the dose to the scalp was kept below 16 Gy in 15 fractions. ${ }^{9}$ In this current study, our two cases with three areas in which recalculation of the dosimetry was possible, repigmentation happened at a mean dose of 16 Gy in 26 fractions equivalent to 0.6 Gy per fraction. Perhaps the mechanism for repigmentation is different to that of depigmentation? Our one case of depigmentation may be explained by the simple destruction of the melanoblasts in the hair follicle bulb by RT. This may have happened at 16 Gy in the 10 fractions delivered in that case. This case was confounded by concurrent melanoma immunotherapy which may have had an impact. The mechanism of action is unknown and further study is needed.

\section{Conclusion}

This case series of hair colour change in the penumbral region of VMAT to the scalp for skin cancer is, to our knowledge, the first report of this phenomenon. Even though rare, the potential for hair colour change may need to be part of the informed consent discussion in patients contemplating this treatment.

\section{Acknowledgments}

The authors wish to thank Aileen Eiszele of A\&L Medical Communications for writing assistance and for preparing the journal submission. They would also like to thank GenesisCare staff and the patients involved in this paper.

\section{Funding sources}

Cancer Australia support through the Priority-driven Collaborative Cancer Research Scheme (PdCCRS/1084046) is gratefully acknowledged for this sub-study.

\section{Conflicts of interest and disclosures}

The authors have no conflicts of interest or disclosures to declare.

\section{References}

1. Farthmann B, Schmitz S, Krasagakis K, et al. Photoprotection by total melanin content and pigment phenotype (eumelanin, pheomelanin) in human melanoma cell Lines. In: Altmeyer P, Hoffmann K, Stücker M, editors. Skin Cancer and UV Radiation. Springer, Berlin, Heidelberg; 1997.
2. Watanabe T, Yagata H, Saito M, et al. A multicenter survey of temporal changes in chemotherapy-induced hair loss in breast cancer patients. PLoS One. 2019;14(1):e0208118.

3. Rivera N, Boada A, Bielsa MI, et al. Hair repigmentation during immunotherapy treatment with an anti-programmed cell death 1 and antiprogrammed cell death ligand 1 agent for lung cancer. JAMA Dermatol. 2017;153(11):1162-1165.

4. Etienne G, Cony-Makhoul P, Mahon FX. Imatinib mesylate and gray hair. N Engl J Med. 2002;347(6):446.

5. Alexander LL. Hair color changes following radiation epilation. J Natl Med Assoc. 1963;55(6):546-548.

6. Potter A, Price M, Papworth D, et al. A technique for treating extended skin field cancerisation using volumetric modulated arc therapy. Int $J$ Radiol Radiat Ther. 2019;6(4):111-119.

7. Daniel Santos E, Green JA, Bhandari N, et al. Tangential volumetric modulated radiotherapy - A new technique for large scalp lesions with a case study in lentigo maligna. Int J Bioauthomation. 2015;19(2):223-236.

8. Alcevski B, Shearer T, Yeong Y, et al. Techniques to verify the correct skin areas for biopsy, treatment, recurrence and in vivo dosimetry using an A4 plastic sheet as template. Int J Radiol Radiat Ther. 2020;7(4):112-118.

9. Williams N, Boyle F, Hong A, et al. Whole brain radiotherapy with partial hair-sparing technique - a feasibility study. Int $J$ Radiol Radiat Ther. 2020;7(4):104-110.

10. Kumar AB, Shamim H, Nagaraju U. Premature graying of hair: Review with updates. Int J Trichology. 2018;10(5):198-203.

11. Slominski A, Paus R. Melanogenesis is coupled to murine anagen: Toward new concepts for the role of melanocytes and the regulation of melanogenesis in hair growth. J Invest Dermatol. 1993;101:90S-97S.

12. Nsikan Akpan. Cure for gray hair is almost here, vitiligo study claims. Medical Daily. 2013.

13. Trüeb RM. Oxidative stress in ageing of hair. Int $J$ Trichology. 2009;1(1):6-14.

14. International Commission on Radiological Protection. Avoidance of radiation injuries from medical intervention procedures. ICRP Publication 85. Ann ICRP. 2000;30:7-67.

15. Hamilton CS, Potten CS, Denham JW, et al. Response of human hair cortical cells to fractionated radiotherapy. Radiother Oncol. 1997;43:289292.

16. Lawenda BD, Gagne HM, Gierga DP, et al. Permanent alopecia after cranial irradiation: dose-response relationship. Int J Radiat Oncol Biol Phys. 2004;60(3):879-887.

17. Severs GA, Griffin T, Werner-Wasik M. Cicatricial alopecia secondary to radiation therapy: case report and review of the literature. Cutis. 2008;81(2):147-153. 